\title{
Classification criteria for mild cognitive impairment
}

\section{A population-based validation study}

\author{
Karen Ritchie, PhD; Sylvaine Artero, MA; and Jacques Touchon, MD
}

\begin{abstract}
Article abstract-Objective: To evaluate the predictive validity and temporal stability of diagnostic criteria for mild cognitive impairment (MCI). Background: MCI has been proposed as a nosologic entity referring to elderly persons with subclinical cognitive deficits due to incipient dementia. Classification criteria, which have been derived from small, selected clinical groups, are currently disputed, and have not yet been assessed within the general population. Methods: Subjects meeting current criteria for MCI and also age-associated cognitive decline (AACD-a similar concept that is assumed to be related to normal cognitive aging processes rather than incipient dementia) were identified within each of three waves of a longitudinal population study, which included a standardized neurologic examination. Results: In the general population, the prevalence of MCI was estimated to be $3.2 \%$ and AACD 19.3\%. MCI was a poor predictor of dementia within a 3 -year period, with an $11.1 \%$ conversion rate. Subjects with MCI also constituted an unstable group, with almost all subjects changing category each year. Discriminant function analysis failed to isolate a homogeneous clinical group. Subjects classified as AACD, contrary to the theoretical assumptions underlying the disorder, represented a more stable group, with a $28.6 \%$ conversion rate to dementia over 3 years (relative risk $=21.2$ ). Conclusion: MCI criteria perform poorly when applied to a representative population sample. The authors propose modifications to current diagnostic criteria to increase their capacity to detect incipient dementia.
\end{abstract}

NEUROLOGY 2001;56:37-42

Cognitive impairment without dementia has been commonly considered to be a normal consequence of brain aging. It has been of interest to clinicians because of the difficulties that it may engender in the performance of everyday activities. A number of clinical labels have been proposed to describe subclinical cognitive deficits. ${ }^{1}$ Earlier concepts such as "benign senescent forgetfulness," "age-associated memory impairment," (AAMI) ${ }^{3}$ "age-associated cognitive decline"(AACD), ${ }^{4}$ and "age-related cognitive decline" ${ }^{5}$ consider such mild cognitive deficits to fall within the limits of normal aging. More recently, the "normality" of these subclinical states has been called into question, because subjects with objectively demonstrated deficits have been shown to be at increased risk for neurodegenerative disease, show quantitative and qualitative differences in cerebral imaging, and share common biologic and environmental risk factors. ${ }^{1}$ A number of concepts have thus been proposed that link subclinical cognitive impairment to pathologic states: mild cognitive disorder, ${ }^{6}$ mild neurocognitive disorder, ${ }^{5}$ and mild cognitive impairment (MCI). ${ }^{7}$

The concept of MCI has predominated in the United States, where large-scale research programs have recently been initiated, with an ultimate view of providing treatment, and therefore reducing the risk of progression to senile dementia. In Europe, reference is still more commonly made to AAMI and AACD—states linked rather to the normal biologic aging process. MCI is probably the more seductive concept for clinicians and researchers because, unlike AAMI and AACD, it is assumed to be pathologybased and therefore amenable to intervention. MCI at a nosologic level remains, however, problematic, and there is still no common consensus on diagnostic criteria.

A principal problem of research in this area is that it has been largely confined to small, selected clinical populations. In many cases, dementia screening tests are applied in the selection process, thus confounding observations of the relationship between MCI and $\mathrm{AD}$. Population studies have largely focused on dementia syndromes, and therefore provide little information on subclinical levels of impairment. In 1991, a study of cognitive functioning in normal elderly individuals recruited from a representative sample of general practices was initiated in the Montpellier region in the south of France. This study, the Eugeria Project, involved the 3-year follow-up of a cohort of subjects with subclinical cognitive deficit, thus constituting a rare general population database on low-level cognitive impairments.

The current study aims to examine the utility and

From the Institut National de la Santé et de la Recherche Médicale, INSERM E99 30 Epidemiology of Nervous System Pathologies, Montpellier, France. The Eugeria Project was supported by the French Social Security (CNAM-TS), the Fondation de France, and the Direction Générale de la Santé. Received April 25, 2000. Accepted in final form September 15, 2000.

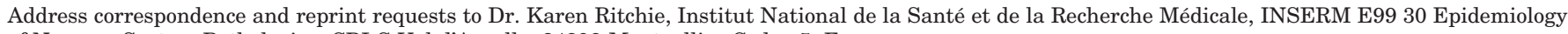
of Nervous System Pathologies, CRLC Val d'Aurelle, 34298 Montpellier Cedex 5, France. 
prognostic value of the concept of MCI by retrospectively identifying the subjects in this study meeting MCI criteria at each wave and examining their clinical outcome and associated characteristics. Previous clinical studies of MCI have suggested that subjects with MCI may have smaller medial temporal lobe volumes, ${ }^{8-11}$ and that risk factors for progression from $\mathrm{MCI}$ to $\mathrm{AD}$ are higher age, the presence of the APOE $\epsilon 4$ allele, fine motor deficit, and lower premorbid IQ. ${ }^{7,12}$ These factors are considered in the current general population validation study. The cognitive tests used to investigate MCI thus far have often been limited to those used to identify $\mathrm{AD}$, or tests of general ability. The current study examines potential cases of MCI using a comprehensive battery of cognitive tests designed to assess all aspects of information processing as it is currently conceptualized by cognitive psychologists. The clinical characteristics and prognostic value of MCI is compared in this study with those of AACD, with the latter representing an alternative formulation based on the underlying theoretical supposition that subclinical cognitive decline is a normal feature of the aging process and not an early pathologic process.

Methods. The subjects for this study are taken from a general practitioner research network created by the research unit in collaboration with the regional medical association as part of the Eugeria longitudinal study of cognitive aging. The network is representative of general practice in the region, covering both urban and rural areas. An intensive training course in psychogeriatric screening and application of criteria for senile dementia according to the Diagnostic and Statistical Manual, 3rd ed., revised ${ }^{13}$ (DSM III-R)was given to the 63 general practitioners in the network. Eight hundred and thirty-three subjects over 60 years of age without senile dementia were recruited into the study in the first year. A proxy screening questionnaire on cognitive functioning over the past year was sent to all subjects. This screening instrument, Détérioration Cognitive Observée (DECO), has been shown in previous studies to be highly sensitive to early changes in cognitive functioning due to various causes. ${ }^{14}$ It is based on the degree of change in cognitive functioning over the last year, as estimated by a proxy who has had at least monthly contact with the subject over the past 3 years. Of these subjects, 397 were found to have a score of less than 38 , with this being the maximum total score. These persons were thus considered by an observer to have shown some degree of observable deterioration in at least one area of cognitive functioning over the past year, and also to have a subjective complaint of declining ability. These subjects were followed over a further 2 years, along with a random sample $(n=73)$ of the remaining subjects without cognitive complaints.

A computerized neuropsychometric examination, Examen Cognitif par Ordinateur (ECO), was given to all 833 subjects in the first year and annually to the subjects followed over 2 more years. ECO assesses primary memory, verbal and visuospatial secondary memory, language skills (word and syntax comprehension, naming, verbal fluency), visuospatial performance (ideational, ideomotor, and con- structive apraxia; functional and semantic categorization of visual data; visual reasoning; and form perception), and focused and divided attention (visual and auditory modalities). The development of ECO and the theoretical basis for test selection is described elsewhere. ${ }^{15}$ Response latencies were recorded using a tactile screen.

From the 159 ECO variables, 10 summary scores representing six cognitive domains were used in the analysis:

Attention: measured by response time on a dual task (simultaneous visual selection and counting of auditory stimuli)

Primary memory: assessed by immediate recall of first names with and without cues which had the highest frequency in the French language 50 years ago

Secondary memory: measured by 1) delayed recall of first names and 2) their associated faces

Visuospatial ability: measured by 1) reference to response time on shape, functional, and semantic visual matching tasks and 2) the number of elements correct in the copying of meaningful and meaningless figures

Language: assessed by 1) mean reaction time on word and syntax comprehension, 2) naming, and 3) verbal fluency using both phonetic and functional cues

Reasoning: assessed by completion of logical visual series

A series of validated scales examining the capacity to perform a wide range of activities of daily living, the Echelle de Comportement et d'Adaptation (ECA) scale, ${ }^{16}$ was completed in collaboration with both subjects and caregivers at each wave of the study. Information was also obtained concerning depressive symptomatology according to DSM III-R criteria. $^{13}$ In the third year, a standardized neurologic examination with SPECT for the diagnosis of psychogeriatric disorder based on DSM III-R ${ }^{13}$ criteria was performed by a neurologist who had no knowledge of the results of the cognitive tests. Blood samples were collected to establish APOE status. A consent form describing the aims and methods of the study was signed by all subjects. Authorization for the study was also obtained from the National Data Protection and Ethics Committee.

At entry into the study (wave 1) and at yearly follow-up (waves 2 and 3), subjects meeting MCI and AACD criteria were identified. The criteria used for MCI initially proposed by Petersen et al. ${ }^{17}$ specify 1) the presence of a subjective memory complaint, 2) preserved general intellectual functioning as estimated by performance on a vocabulary test, 3 ) demonstration of a memory impairment by cognitive testing, 4) intact ability to perform activities of daily living, and 5) absence of dementia. More recently, the same authors have stipulated that there should be impairment on a memory task only and not on tests relating to other cognitive functions. ${ }^{7}$ Criteria for AACD are consistent with previously established consensus guidelines $^{4}$ of decline of more than one SD in any area of cognitive functioning in comparison with age-matched controls.

Results. Among the 833 subjects recruited into the study, 308 subjects were identified based on MCI criteria 1 and 2. These were all included in the cohort of 397 subjects followed longitudinally. Of these, 103 subjects demonstrated a decrement of more than one SD on a memory task compared with ECO standardization data matched by age and level of education (MCI criterion 3). Applying the additional criterion, which excludes subjects with a diffi- 
Table 1 Classification of subjects with mild cognitive impairment (MCI) and age-associated cognitive decline (AACD) across three waves of the study

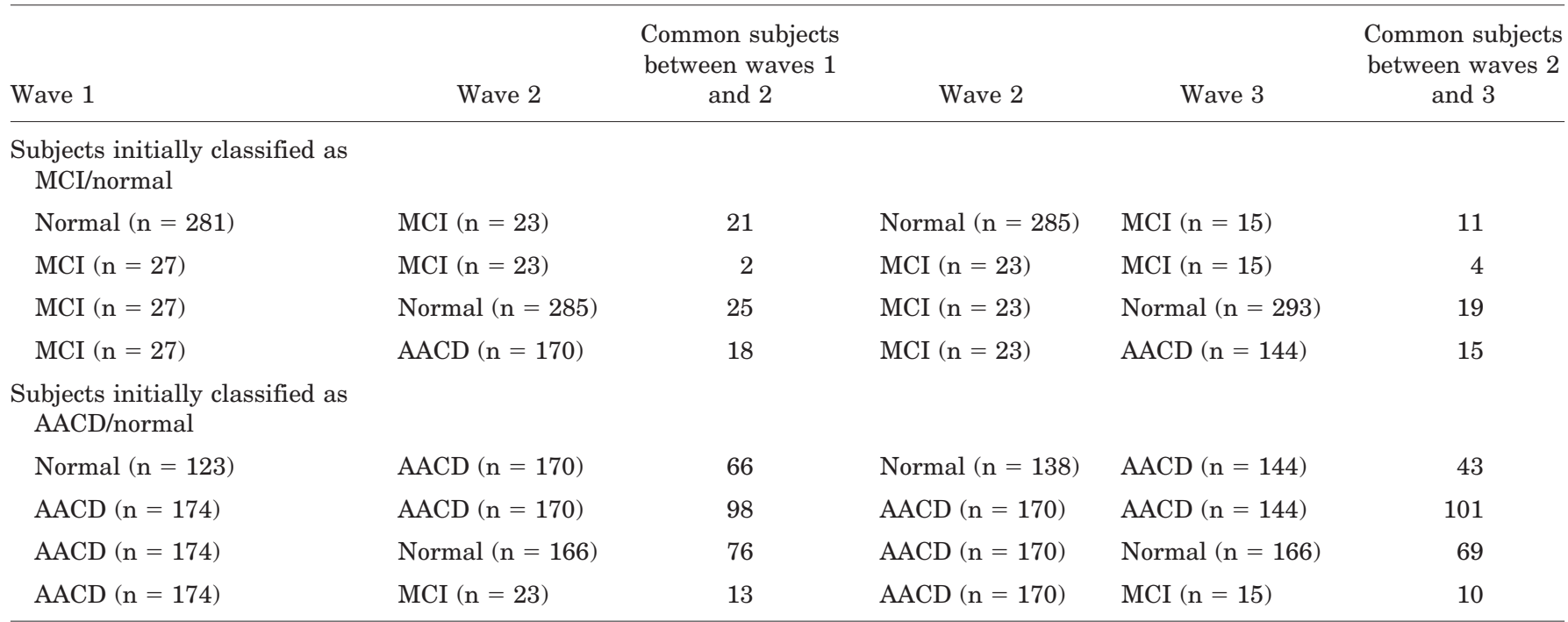

culty in any other area of cognitive functioning, led to the exclusion of all but 27 subjects. The application of MCI criterion 4 led to the inclusion of all remaining subjects, with a definition of disability as "persistent inability to perform activities of daily living without assistance." All subjects classified as MCI or AACD were included in the follow-up.

Twenty-seven subjects were thus classified as MCI in wave 1,23 in wave 2 , and 15 in wave 3 . One hundred and seventy-four were classified as AACD in wave 1, 170 in wave 2 , and 144 in wave 3 . The prevalence of MCI in the general population as estimated from the baseline examinations of all 833 subjects is $3.24 \%$. The prevalence of AACD is $20.9 \%$. Within the cognitive complaint cohort followed over 2 additional years, the prevalence of MCI is estimated to range from 6.8 to $8.5 \%$, and AACD from 48.7 to $56.8 \%$.

All persons classified as MCI were included in the AACD group in each wave, thus precluding the use of discriminant function analysis or similar methods to discriminate the characteristics of the two groups. The stability of each nosologic entity was then examined across time by examining the subjects originally classified as MCI who remained in this group during subsequent waves of the study (table 1). It can be seen that of the 27 subjects classified as MCI in wave 1, only two (7.4\%) retained this diagnosis in wave 2 . Twenty-one new incident cases of MCI appeared in wave 2, of whom only four (17.4\%) are still considered to have MCI in wave 3.

Changes across time in group membership are also seen in subjects classified as AACD. Of the 174 cases identified in wave $1,98(56.3 \%)$ were again given this status in wave 2. Of the 170 subjects classified as AACD in wave 2, 101 $(59.4 \%)$ were still in this group in wave 3 . None of the subjects classified as MCI in wave 1 and only $11.1 \%$ of those who were classified as MCI in wave 2 received a diagnosis of senile dementia by wave 3 . Chi-square values were not significant. Conversely, $17.5 \%$ of the subjects classified as AACD in wave 1 and $28.6 \%$ in wave 2 had a diagnosis of dementia by wave 3 . Prediction of senile dementia across time to diagnosis as evaluated by logistic regression suggests only AACD to be a significant determinant of dementia (relative risk $=21.2$ ) at wave 2. Receiver operating characteristics analysis has been used to evaluate the relative predictive power of MCI and AACD as diagnosed in wave 1 to predict dementia in the following 2 years (figure, table 2).

The curve lying along the diagonal indicates a total inability for the MCI diagnosis to predict dementia status (area under the curve [AUC] $=0.485 ; p=0.842$ ), whereas the curve for the AACD group, rising high above the diagonal to the left, is indicative of high discrimination (AUC = $0.744 ; p=0.001$ ). The mean summary cognitive test scores for the MCI and AACD groups in the second year of the

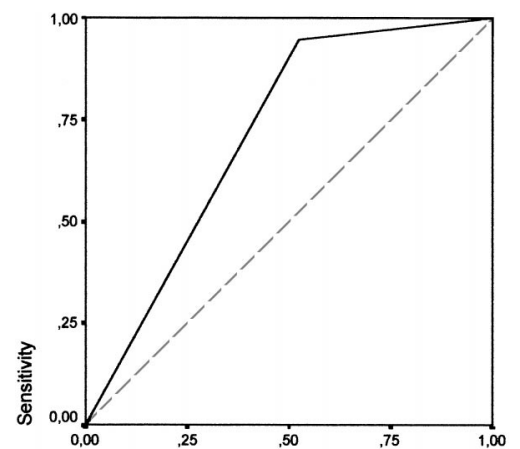

A false positives (1 - Specificity)

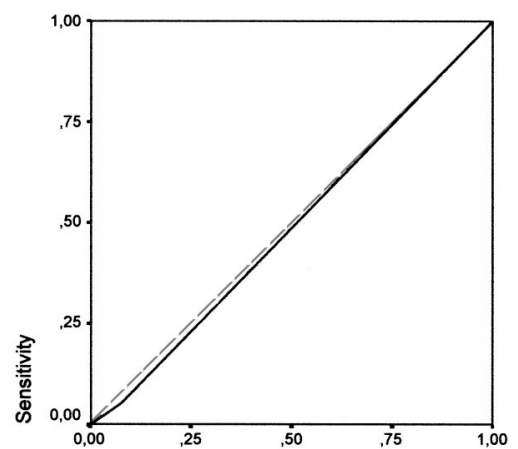

B false positives (1 - Specificity)
Figure. Receiver operating characteristic (ROC) curves showing the ability of age-associated cognitive decline and mild cognitive impairment criteria to diagnose dementia. (A) ROC curve for ageassociated cognitive decline. (B) ROC curve for mild cognitive impairment. 
Table 2 Receiver operating characteristics analysis of the discriminability of AACD and MCI for the prediction of dementia after 2 years

\begin{tabular}{|c|c|c|c|c|c|c|}
\hline Classification & AUC & $\mathrm{SE}$ & $\begin{array}{l}\text { Asymptotic } \\
\text { significance }\end{array}$ & $95 \% \mathrm{CI}$ & Sensitivity, \% & Specificity, \% \\
\hline AACD & 0.744 & 0.051 & 0.001 & $0.644-0.844$ & 94.73 & 54.08 \\
\hline
\end{tabular}

AACD = age-associated cognitive decline; $\mathrm{MCI}=$ mild cognitive impairment; $\mathrm{AUC}=$ area under the curve.

study (the point of highest predictive validity) are given in table 3.

AACD, MCI, and normal groups were compared on the following variables: age, sex, education, SPECT hypoperfusion in eight cortical regions and overall hypoperfusion index, DECO score, family history of cognitive impairment, medication use, exposure to anesthesia, illness over the past year, depressive illness, and APOE status. Discriminant function analysis was also used to compare the MCI and AACD groups with normal subjects. No significant difference was found between the groups on any of these variables either on univariate analysis or by discriminate function analysis. Two subjects with AACD showed significantly higher frontotemporal hypofusion on SPECT.

Discussion. We sought to determine whether MCI may be considered to constitute a separate, homogeneous syndrome. In his discussion of clinical validity in psychiatry, Kendell ${ }^{18}$ has stipulated that such an entity should meet two criteria: 1 ) there should be a cluster of related symptoms with a characteristic time course as demonstrated by clinical observation or cluster analysis; and 2) boundaries between the new syndrome and related syndromes should be demonstrated by discriminant function analysis. Application of currently used MCI criteria to a general population sample has shown that this clinical entity does not meet these criteria for the existence of a homogeneous syndrome, showing neither temporal stability nor clear boundaries from normal subjects apart from the cognitive deficit which defines it. MCI also has poor predictive validity for the onset of dementia within the general population. Conversely, AACD subjects appear to constitute a relatively stable group, with a high predictive validity for dementia onset. This, however, is contradictory to the underlying conceptual basis of AACD as a benign, stable impairment related to the normal aging process and unrelated to the pathologic processes of the senile dementias.

The study does confirm, however, that complaints of low-grade cognitive impairment verified by neuropsychological assessment are not benign and should not be dismissed as a normal feature of aging. Within our cognitive complaint cohort followed over 3 years, the conversion rate to senile dementia ( $18 \%$ incidence over 3 years) is much higher than that observed in the general population. Our results are in agreement with previous clinical observations of subclinical cognitive deficit, ${ }^{7-10,19}$ which report conversion rates of 15 to $53 \%$ over a similar time period. MCI criteria as currently defined have not, however, adequately captured this prodromal group in the general population. This may be due in part to differences in the neuropsychological tests used. At present, no specific tests have been stipulated, and this is a major shortcoming of current MCI criteria. Test differences are not, however, sufficient to explain the high rates of instability observed in our study, as our previous validation study of the ECO battery demonstrates high retest reliability for all tests. Furthermore, a high predictive value of AACD for dementia was found using the same battery, suggesting that a high-risk dementia group may be identified in the general population by means of the cognitive tests used in this study. Our findings suggest that certain modifications to current MCI criteria may greatly increase its predictive value.

Firstly, because the number of MCI cases found is small, and all are included within the AACD group, it would appear that current criteria for MCI are too stringent. The principal difference between MCI and AACD lies in the prerequisite that there be impairment in memory, but not in any other area of cognitive functioning. This point has already been the subject of much debate, as an increasing number of studies conclude that subjects with MCI, although having primarily memory complaints, also commonly show deficits on tasks of language, ${ }^{12,20}$ orientation, ${ }^{10}$ and praxis..$^{12,20}$ Although there is some evidence that a purely mnesic syndrome may exist within a clinical context, ${ }^{7}$ this appears to be a rare occurrence when the full range of cognitive functions are examined. ${ }^{21}$ Adhering to the strict criteria of isolated memory complaint in this study would have led to unacceptable levels of sensitivity, with the number of cases of isolated deficit falling well below the expected prevalence of $\mathrm{AD}$ itself. The isolation of a pure mnesic syndrome based on neuropsychometric testing methods is in itself highly questionable; even the so-called memory tests involve cognitive functions other than memory.

Comparative studies of MCI would be greatly facilitated if standardized cognitive testing procedures could be specified. The current study has used a much broader battery of cognitive tests than those used to date in current clinical studies, therefore providing information on the tests that are most likely to be sensitive to early dementia 1 and 2 years before diagnosis. From a previous analysis of the Eugeria data, ${ }^{22}$ we established that the following tests were able to differentiate normal subjects from those with preclinical senile dementia 2 years before 


\begin{tabular}{|c|c|c|c|c|}
\hline \multirow[b]{2}{*}{ Task } & \multicolumn{4}{|c|}{ Group } \\
\hline & $\begin{array}{l}\text { Normal } \\
(\mathrm{n}=64)\end{array}$ & $\begin{array}{c}\text { MCI } \\
(\mathrm{n}=23)\end{array}$ & $\begin{array}{c}\text { AACD } \\
(\mathrm{n}=170)\end{array}$ & $\begin{array}{c}\text { Dementia } \\
(\mathrm{n}=19)\end{array}$ \\
\hline \multicolumn{5}{|l|}{ Attention } \\
\hline Mean reaction time on double task & $19.94(4.49)$ & $21.03(4.0)$ & $25.42(7.85)$ & $31.43(7.04)$ \\
\hline \multicolumn{5}{|l|}{ Primary memory } \\
\hline \multicolumn{5}{|l|}{ Secondary memory } \\
\hline Delayed recall of names & $5.48(1.80)$ & $3.0(1.73)$ & $3.37(2.17)$ & $2.57(2.38)$ \\
\hline Delayed recall of faces & $7.96(1.24)$ & $7.30(1.74)$ & $6.77(1.99)$ & $5.84(2.11)$ \\
\hline \multicolumn{5}{|l|}{ Visuospatial ability } \\
\hline Naming total correct & $9.54(0.68)$ & $9.8(0.38)$ & $8.55(1.68)$ & $7.52(1.71)$ \\
\hline Fluency total & $39.01(10.98)$ & $32.21(7.76)$ & $25.32(11.33)$ & $18.57(8.02)$ \\
\hline Reaction time word and syntax comprehension & $17.81(4.92)$ & $19.05(4.87)$ & $26.56(10.16)$ & $33.16(13.64)$ \\
\hline \multicolumn{5}{|l|}{ Reasoning } \\
\hline Logical series total correct & $1.45(1.09)$ & $1.52(0.51)$ & $0.76(0.89)$ & $0.42(0.50)$ \\
\hline
\end{tabular}

Values expressed as mean (SD).

MCI = mild cogitive impairment; AACD = age-associated cognitive decline

diagnosis, and were also independent of education effects: simple reaction time, reaction time on a dual attention task, semantic category fluency, delayed free verbal recall, cued delayed verbal recall, recall of name-face pairs, narrative recall, and copying of a complex design. This concurs with previous studies, which have suggested delayed free and cued recall to be the most predictive of dementia onset, ${ }^{7,17}$ but also indicates that other tests may be useful in improving predictive validity.

The second difference between MCI and AACD criteria relates to performance of activities of everyday living. No guidelines have been given as to what constitutes activities of daily living restriction in MCI. Any number of activities might be proposed, which may be more or less culturally biased. The degree of disability is also not stipulated. This study is based on a definition of disability as persistent loss of ability to perform certain everyday activities. However, in previous analyses, it has been shown that very slight changes in activity performance (e.g., occasionally requiring assistance or needing to be reminded to perform an activity) are commonly observed in incipient $\mathrm{AD}$ up to 2 years before diagnosis. $^{22}$ This suggests that activity changes may be seen in MCI if lower thresholds are used to define restriction. This difficulty has been overcome in studies using Clinical Dementia Rating 0.5 classification to define $\mathrm{MCI},{ }^{23}$ as this criterion refers to "usually intact" activities of daily living. ${ }^{19}$ We suggest that, in refining criteria for the identification of
MCI in the general population, the predictive validity of introducing some mild difficulties in activity performance as positive inclusion criteria should also be examined.

\section{References}

1. Ritchie K, Touchon J. Mild Cognitive impairment: conceptual basis and current nosological status. Lancet 2000;335:225228.

2. Kral VA. Senescent forgetfulness: benign and malignant. Can Med Assoc J 1962;86:257-260.

3. Crook T, Bartus RT, Ferris SH, et al. Age associated memory impairment: proposed diagnostic criteria and measures of clinical change: report of a National Institute of Mental Health Work Group. Dev Neuropsychol 1986;2:261-276.

4. Levy R, Aging-associated cognitive decline. Int Psychogeriatr 1994;6:63-68.

5. American Psychiatric Association. Diagnostic and statistical manual, 4th ed. Washington, DC: American Psychiatric Association, 1994.

6. World Health Organization. The ICD-10 classification of mental and behavioural disorders. Diagnostic criteria for research. Geneva, Switzerland: World Health Organization, 1993.

7. Petersen RC, Smith GE, Waring SC, et al. Mild cognitive impairment: clinical characterization and outcome. Arch Neurol 1999;56:303-308.

8. Johnson KA, Jones K, Holman BL. Preclinical prediction of Alzheimer's disease using SPECT. Neurology 1998;50:15631572.

9. Black SE. Can SPECT predict the future for mild cognitive impairment ? Can J Neurol Sci 1999;26:4-6.

10. Wolf H, Grunwald M, Ecke GM, et al. The prognosis of mild cognitive impairment in the elderly. J Neural Transm 1998; 54:31-50.

11. Krasuki JS, Alexander GE, Horwitz B, et al. Volumes of medial temporal lobe structures in patients with Alzheimer's 
disease and mild cognitive impairment (and in healthy controls). Biol Psychiatry 1998;43:60-68.

12. Kluger A, Gianutsos JG, Golomb J, et al. Motor/psychomotor dysfunction in normal aging, mild cognitive decline, and early Alzheimer's disease: diagnostic and differential diagnostic features. Int Psychogeriatr 1997;9:307-316.

13. American Psychiatric Association. Diagnostic and statistical manual. 3rd ed., revised. Washington, DC: American Psychiatric Association, 1987.

14. Ritchie K, Fuhrer R. A comparative study of the performance of screening tests for senile dementia using receiver operating characteristics analysis. J Clin Epidemiol 1992;45:627-637.

15. Ritchie K, Allard M, Huppert FA, et al. Computerized cognitive examination of the elderly (ECO): the development of a neuropsychological examination for clinic and population use. Int J Geriatr Psychiatry 1993;8:899-914.

16. Ritchie K, Ledésert B. The measurement of incapacity in the severely demented elderly: the validation of a behavioural assessment scale. Int J Geriatr Psychiatry 1991;6:217-226.

17. Petersen RC, Smith GE, Waring SC, et al. Aging memory and mild cognitive impairment. Int Psychogeriatr 1997;9:65-69.

18. Kendell RE. Clinical validity. Psychol Med 1989;19:45-55.

19. Rubin EH, Morris JC, Grant EA, et al. Very mild senile dementia of the Alzheimer type. I Clinical assessment. Arch Neurol 1989;46:379-382.

20. Flicker C, Ferris FH, Reisberg B. Mild cognitive impairment in the elderly: predictors of dementia. Neurology 1991;41: 1006-1009.

21. Richards M, Touchon J, Ledésert B, et al. Cognitive decline in ageing: are AAMI and AACD distinct entities? Int J Geriatr Psychiatry 1999;14:534-540.

22. Touchon J, Ritchie K. Prodromal cognitive disorder in Alzheimer's disease. Int J Geriatr Psychiatry 1999;14:556-563.

23. Hughes CP, Berg L, Danziger WL, et al. A new scale for the staging of dementia. Br J Psychiatry 1982;140:566-535.

\title{
сме Cardiovascular risk factors and cognitive decline in middle-aged adults
}

\author{
D. Knopman, MD; L.L. Boland, MPH; T. Mosley, PhD; G. Howard, DrPH; D. Liao, MD, PhD; \\ M. Szklo, MD, DrPH; P. McGovern, MD; and A.R. Folsom, MD, for the \\ Atherosclerosis Risk in Communities (ARIC) Study Investigators
}

Article abstract-Objective: To perform serial neuropsychological assessments to detect vascular risk factors for cognitive decline in the Atherosclerosis Risk in Communities cohort, a large biracial, multisite, longitudinal investigation of initially middle-aged individuals. Methods: The authors administered cognitive assessments to 10,963 individuals (8,729 white individuals and 2,234 black individuals) on two occasions separated by 6 years. Subjects ranged in age at the first assessment from 47 to 70 years. The cognitive assessments included the delayed word recall (DWR) test, a 10-word delayed free recall task in which the learning phase included sentence generation with the study words, the digit symbol subtest (DSS) of the Wechsler Adult Intelligence Scale-Revised and the first-letter word fluency (WF) test using letters F, A, and S. Results: In multivariate analyses (controlling for demographic factors), the presence of diabetes at baseline was associated with greater decline in scores on both the DSS and WF $(p<0.05)$, and the presence of hypertension at baseline was associated with greater decline on the DSS alone $(p<0.05)$. The association of diabetes with cognitive decline persisted when analysis was restricted to the 47- to 57-year-old subgroup. Smoking status, carotid intima-media wall thickness, and hyperlipidemia at baseline were not associated with change in cognitive test scores. Conclusions: Hypertension and diabetes mellitus were positively associated with cognitive decline over 6 years in this late middle-aged population. Interventions aimed at hypertension or diabetes that begin before age 60 might lessen the burden of cognitive impairment in later life.

NEUROLOGY 2001;56:42-48

Vascular risk factors are typically thought to produce deleterious effects on brain function via overt strokes. However, it is more likely that the burden of brain lesions due to cerebrovascular disease actually accumulates subclinically over years or decades. Cross-sectional data in elderly demented individuals have shown that various cardiovascular risk factors are associated with a higher risk of dementia. ${ }^{1}$ How early these risk factors may begin to exert their influence is not well understood. Several studies have noted that blood pressure during middle age is associated with later cognitive dysfunction. ${ }^{2-8}$ None of

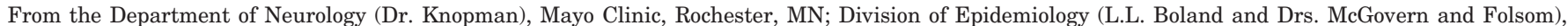

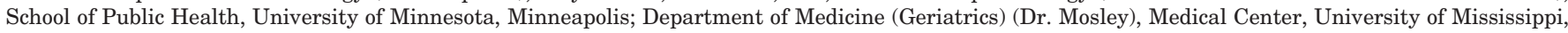

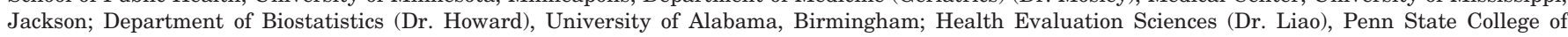
Medicine, Hershey, PA; and Department of Epidemiology (Dr. Szklo), The Johns Hopkins Medical Institutions, Baltimore, MD.

Supported by contracts N01-55015, N01-55016, N01-55018, N01-55019, N01-55020, N01-55021, N01-55022 from the National Heart, Lung and Blood Institute.

Presented at the annual meeting of the American Academy of Neurology, San Diego, CA; May 3, 2000.

Received May 18, 2000. Accepted in final form September 21, 2000.

Address correspondence and reprint requests to Dr. David Knopman, Department of Neurology, Mayo Clinic, 200 First Street SW, Rochester, MN 55905. 


\section{Neurology}

\section{Classification criteria for mild cognitive impairment: A population-based validation study}

Karen Ritchie, Sylvaine Artero and Jacques Touchon

Neurology 2001;56;37-42

DOI 10.1212/WNL.56.1.37

\section{This information is current as of January 9, 2001}

\section{Updated Information \&} Services

References

Citations

Subspecialty Collections

Permissions \& Licensing

Reprints including high resolution figures, can be found at: http://n.neurology.org/content/56/1/37.full

This article cites 16 articles, 3 of which you can access for free at: http://n.neurology.org/content/56/1/37.full\#ref-list-1

This article has been cited by 41 HighWire-hosted articles: http://n.neurology.org/content/56/1/37.full\#\#otherarticles

This article, along with others on similar topics, appears in the following collection(s):

All Cognitive Disorders/Dementia

http://n.neurology.org/cgi/collection/all_cognitive_disorders_dementia MCI (mild cognitive impairment)

http://n.neurology.org/cgi/collection/mci_mild_cognitive_impairment

Information about reproducing this article in parts (figures,tables) or in its entirety can be found online at:

http://www.neurology.org/about/about_the_journal\#permissions

Information about ordering reprints can be found online:

http://n.neurology.org/subscribers/advertise

Neurology ${ }^{\circledR}$ is the official journal of the American Academy of Neurology. Published continuously since 1951, it is now a weekly with 48 issues per year. Copyright . All rights reserved. Print ISSN: 0028-3878. Online ISSN: 1526-632X.

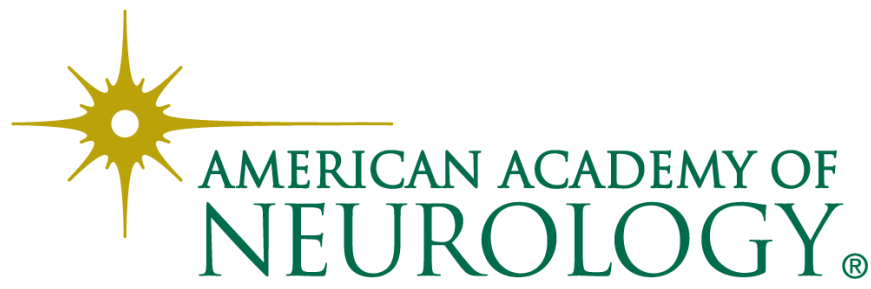

